\title{
ON THE GROUPS GENERATED BY TWO OPERATORS.
}

BY DR. G. A. MILLER.

(Read before the American Mathematical Society, April 27, 1901.)

IT is well known that the only abelian groups which are generated by two operators are those which have just two independent generators.* If the order of such a group is $p^{m}$ ( $p$ being any prime number), it must be of type $(m-\alpha, \alpha)$. In general, if the order of any one of these abelian groups

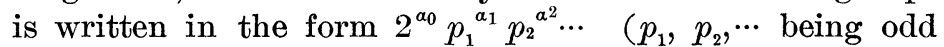
prime numbers) it is clear that at least one of the subgroups of orders $2^{a_{0}}, p_{1}^{a_{1}}, p_{2}^{a_{2}}, \cdots$ contains just two independent generators while the remaining ones (if any) are cyclic.

When the two generating operators are not commutative the matter becomes much more difficult. A very interesting and simple case presents itself when each of these operators $\left(s_{1}, s_{2}\right)$ is of order two. From the equation $s_{1} s_{2}=s_{3}$ we obtain the following :

$$
s_{2}=s_{1} s_{3} \quad s_{2} s_{1}=s_{1} s_{3} s_{1}=\left(s_{1} s_{2}\right)^{-1}=s_{3}^{-1}=s_{2} s_{3} s_{2} .
$$

Since $s_{1}$ and $s_{2}$ transform $s_{3}$ into its inverse they generate the group of dihedral rotations. $\dagger$ By properly selecting $s_{1}$ and $s_{2}$ the order of $s_{3}$ can be made any positive integer whatever. $\$$ That is, every group that is generated by two operators of order two is a dihedral rotation group and every dihedral rotation group is generated by two operators of order two. When the order of $s_{3}$ is two this group is abelian and vice versa.

From the known theorems which we proceed to give, it follows almost directly that every symmetric group and every alternating group is generated by two operators. (1) If a primitive group whose degree exceeds 8 contains a substitution whose degree is less than 6 , it is alternating or symmetric. $\$$ (2) If a transitive group of degree $n$ contains a substitution whose order is a prime number $p>n / \alpha(\alpha$ being the smallest factor of $n$ ) it is primitive. (3) If two

* Frobenius and Stickelberger, Crelle, vol. 86, 1879, p. 217. Only noncyclic groups are considered in the present note.

† Cf. Klein, Ikosaeder, 1884, p. 9.

$\ddagger$ American Journal of Mathematics, vol. 22, 1900, p. 185.

8 Netto-Cole, Theory of substitutions, 1892, p. 138 ; Bulletin, vol. 4, 1898, p. 141. 
substitutions have only one common element, their commutator is of degree three, and if they have two consecutive elements in common, without being commutative, their commutator is of degree four and order two.* (4) There is at least one prime number between $m$ and $2 m-2$, whenever $m>7 / 2$. $\dagger$

As a direct consequence of the last theorem we have the theorem : The alternating group $G$ of degree $n$ contains a substitution $S_{1}$ of prime order $p>n / 2$. From the other three theorems it follows that $S_{1}$ and any circular substitution $S_{2}$ which involves all the elements of $G$ that are not contained in $S_{1}$ together with either one or two elements of $S_{1}$, generate the symmetric group when $S_{2}$ is negative and the alternating group when $S_{2}$ is positive. If $S_{2}$ is positive when it has one element in common with $S_{1}$, it must be negative when it has two elements in common. Hence every symmetric and every alternating group whose degree exceeds 8 is generated by two operators. It is easy to verify that this theorem is also true when $n \equiv 8+$.

It has been observed that two operators of order two generate a group which is well known. The next case in the order of simplicity presents itself when one of the generating operators $S_{1}$ is of order three while the other $S_{2}$ is of order two. When their product is of order two, three, four, or five, these operators generate the symmetric group of order 6 , the alternating group of order 12 , the symmetric group of order 24, or the alternating group of 60 respectively.§ We proceed to prove that $S_{1}$ and $S_{2}$ may be so chosen that they generate the alternating group and also so that they generate the symmetric group of every degree $n>8$. Let

$s_{1}=a_{1} \alpha_{2} \alpha_{3} \cdot a_{4} a_{5} \alpha_{6} \cdots \cdot a_{\beta-2} a_{\beta-1} a_{\beta}, s_{2}{ }^{\prime}=a_{3} a_{4} \cdot a_{6} a_{7} \cdot \cdots \cdot a_{\beta-3} a_{\beta-2}$.

It is clear that $s_{1} s_{2}{ }^{\prime}$ is a circular substitution of degree $\beta$. We shall form $s_{2}$ by adding suitable transpositions to $s_{2}{ }^{\prime}$. In what follows $p=n-\alpha$ will be used to represent a prime number such that $n-2>p>\frac{n}{2}$ and it will be assumed that $n>11$ unless the contrary is stated.

When $n=\beta$ and $\alpha$ is odd, we may obtain generators of the alternating and the symmetric group by multiplying $s_{2}^{\prime}$ by $a_{1} a_{\frac{3 \alpha+1}{2}}$ and $a_{1} a_{\frac{3 \alpha-5}{2}} \cdot a_{n-4} a_{n-1}$ respectively ; when $\alpha$ is even, the

* Bochert, Math. Annalen, vol. 40, 1892, p. 176.

†'Tchebichef, Pétersb. Mem., vol. 6 (1851); Liouville, vol. 17 (1852).

\$ Cf. Netto, loc. cit., p. 90.

Z Cf. Burnside, Theory of Groups, 1897, p. 291. 
corresponding multipliers are $a_{1} a_{\frac{3 a-2}{2}} \cdot a_{\beta-1} a_{\beta}$ and $a_{2} \frac{a_{3 a+4}}{2}$ respectively. By using the $s_{2}$ thus obtained and the given $s_{1}$, we can therefore generate any alternating or symmetric group whose degree exceeds 9 and is divisible by 3 . When $n=\beta+1$ and $\alpha$ is odd, we may multiply $s_{2}{ }^{\prime}$ by $\frac{a_{1} \alpha_{3 \alpha+1}}{2} \cdot a_{\beta} a_{\beta+1}$ and $a_{1} a_{3 a-5} \cdot a_{\beta} a_{\beta+1} \cdot a_{\beta-4} a_{\beta-1}$ respectively to obtain the required $s_{2}$; while the corresponding multipliers are $\alpha_{1} \alpha_{\frac{3 \alpha_{-2}}{2}}$. $a_{\beta-4} a_{\beta+1} \cdot a_{\beta-1} a_{\beta}$ and $\alpha_{1} a_{\beta+1} \cdot a_{2} a_{3 \alpha+4}$ when $\alpha$ is even.* Finally, when $n=\beta+2$ and $\alpha$ is odd, suitable factors of $s_{2}^{\prime}$ are $a_{1} a_{\frac{3 a+1}{2}} \cdot a_{\beta-1} a_{\beta+1} \cdot a_{\beta} a_{\beta+2}$ and $a_{1} a_{\frac{3 \alpha-5}{2}} \cdot a_{\beta-7} a_{\beta-4} \cdot a_{\beta-1} a_{\beta+1} \cdot a_{\beta} a_{\beta+2}$; when $\alpha$ is even the factors are $\frac{a_{1} a_{3 \alpha-2}}{2} \cdot a_{\beta-7} a_{\beta+2} \cdot a_{\beta-4} a_{\beta+1} \cdot a_{\beta-1} a_{\beta}$ and $\alpha_{1} \alpha_{\beta+1} \cdot a_{2} a_{3 a+4} \cdot a_{\beta} \alpha_{\beta+2}$, respectively.

When $n<12^{2}$, it is easy to examine the cases directly and thus prove that every alternating group with the exception of those of degrees $3,6,7,8$ is generated by two of its substitutions of orders two and three respectively, and every symmetric group with the exception of those of degrees $5,6,8$ is generated by two of its substitutions of the same orders. Hence 6 and 8 are the only degrees for which neither the symmetric nor the alternating group is generated by two of its substitutions of orders two and three respectively. The only transitive groups generated in these two cases are of orders $6,12,18,24,48,60$, 168 and 336.

\section{A CURIOUS APPROXIMATE CONSTRUCTION FOR $\pi$.}

BY MR. GEORGE PEIRCE.

(Read before the American Mathematical Society, April 27, 1901.)

Construction : $A C, B D$ are perpendicular diameters of a circle. $D E=A O$. $C E$ produced cuts $B D$ in $G$ and the circle in $F$.

To prove that $\pi r=F C+B G$ approximately.

* When $n=13$ we may assume $a=2$, Quar. Jour. of Math., vol. 29 (1898), p. 228 . 